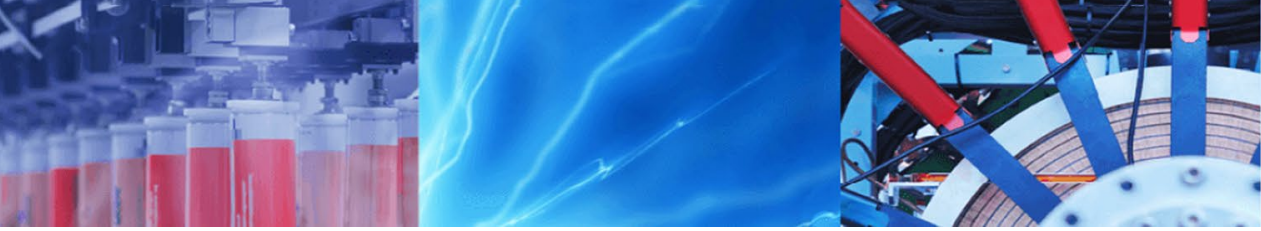

Research Article

\title{
Low-cost and simple FDM-based 3D-printed microfluidic device for the synthesis of metallic core-shell nanoparticles
}

\author{
Lucas P. Bressan ${ }^{1}$ - Taíssa M. Lima ${ }^{1}$ - Géssica D. da Silveira ${ }^{1}$ · José A. F. da Silva ${ }^{1}$ (D)
}

Received: 22 November 2019 / Accepted: 15 April 2020 / Published online: 29 April 2020

(c) Springer Nature Switzerland AG 2020

\begin{abstract}
A fused deposition modeling-based 3D-printed microfluidic device is designed and built to synthesize core-shell metallic Au@Ag nanoparticles based on the reduction of a silver salt on gold nanoparticles seeds by sodium borohydride in a continuous flow manner. A microfluidic device consisting of three inlets for the reactants and one outlet to collect the synthesized nanoparticles is printed in less than two hours using poly(lactic acid) as the polymer, presenting channels width and height of approximately $260 \mu \mathrm{m}$. After synthesis, the nanoparticles samples were analyzed by UV-Vis spectroscopy and transmission electron microscopy to confirm the formation of the core-shell structure. The concentration of the silver salt was varied, while the flow rate was kept constant, and the results indicated that too high silver concentration in relation to amount of gold seeds could lead to coalescence of the synthesized nanoparticles. We obtained core-shell nanoparticles of approximately $23 \mathrm{~nm}$ in diameter. Finally, we employed the synthesized Au@Ag nanoparticles in the surface modification of a carbon paste electrode, which showed improved charge transfer behavior compared to the unmodified electrode against a ferri-ferrocyanide probe, while working as a functionalized electrode in the sensing of thiocyanate ions.
\end{abstract}

Keywords Core-shell $\cdot$ Nanoparticles $\cdot$ FDM-based 3D-printing $\cdot$ Electrochemistry

\section{Introduction}

Microfluidics has been extensively used to synthesize nanoparticles because it enables the fine control of the parameters used in the reactions, allowing for better size and shape uniformity [1-6]. However, during the synthesis of nanoparticles fouling can occur, in which synthesized materials adhere to the walls of the microchannels, therefore requiring their cleansing or discarding [7]. This arises as a problem because the cost of the microfluidic system is strongly linked to the fabrication process, where the devices are usually made of glass, silicon, polydimethylsiloxane (PDMS) or a combination of these materials [8]. To circumvent this, techniques such as flow focusing or segmented flow are used to prepare the nanoparticles, which often requires the use of extra reactants and complex fabrication processes $[9,10]$.

In light of alternative fabrication techniques for lowcost microfluidic devices, fusion deposition modeling (FDM) 3D-printing emerges as a powerful for the manufacturing of complex microchip in a matter of minutes [11]. It employs low-cost polymeric materials, such as acrylonitrile butadiene styrene (ABS) or poly(lactic acid) (PLA), allowing the device to be replaced by an identical without detriments to the synthesis or laboratory budget, if any precipitation is to occur or there is need to replace the microfluidic device [12]. Although FDM presents the lower cost among different 3D-printing techniques, it is

Electronic supplementary material The online version of this article (https://doi.org/10.1007/s42452-020-2768-2) contains supplementary material, which is available to authorized users.

José A. F. da Silva, fracassi@unicamp.br | ${ }^{1}$ Chemistry Institute, State University of Campinas, Campinas, SP 13083-861, Brazil. 
still difficult to print features or microchannels narrow than $100 \mu \mathrm{m}$. However, this can be accomplished by a careful selection of printing parameters and materials, as shown recently by our group [13] and Nelson et al. [14], opening up space to several other applications.

Core-shell nanoparticles present advantages compared to single material ones due to enhanced combination of two or more materials, leading to different optical, electrical and plasmonic properties [15]. They can be made of metallic, non-metallic or a combination of both materials, based on the property one aims to achieve. Despite commonly synthesized in a batch environment, core-shell nanoparticles can also be synthesized in a microfluidic environment, allowing a fine control of the synthetic conditions, such as flow rate, temperature and reagent proportions [15-18].

We already demonstrated the application of this technique in the synthesis of Au and Ag metallic nanoparticles with good results [2]. However, core-shell nanoparticles have not been synthesized using FDM-based microfluidic devices, although they have been extensively researched using more conventional microfluidic materials [19-22]. Specifically, core-shell metallic Au@Ag nanoparticles have been synthesized before in glass microchips, which are laborious to build and are subject to fouling [23].

FDM-based 3D-printed microfluidic devices have been applied to synthetic chemistry recently [24,25], as an alternative to batch protocols in chemistry laboratories that not necessarily have PDMS molding technology available. Here, we suggest using a simple-to-implement, easy-tolearn and fast-to-create technology (3D-printing) to produce microfluidic devices that can be applied to complex, multi-step reactions in a controlled fluidic environment. As highlighted above, these devices are made of less expensive material and do not require multi-step fabrication protocols.

Herein, we demonstrate that the synthesis of core-shell Au@Ag nanoparticles can be achieved in such FDM-based 3D-printed microfluidic device, consisting of channels with length and width as low as $260 \mu \mathrm{m}$, printed in less than two hours and costing less than 0.10 US dollars per device. We carried the synthesis of gold nanoparticles (AuNPs) seeds and then used them to create core-shell Au@Ag nanoparticles. The as-prepared Au@Ag nanoparticles were used to modify a carbon paste electrode (CPE), which was characterized using hexacyanoferrate probe and applied on the measure thiocyanate ions. To the best of our knowledge, this is the first time core-shell nanoparticles are synthesized in an FDM-based 3D-printed microfluidic device.

\section{Materials and methods}

\subsection{Reagents and solutions}

All reagents were used as received. We used tetrachloroauric(III) acid solution $30 \mathrm{wt} \%$ (Sigma-Aldrich, Steinheim, Germany), trisodium citrate (Sigma-Aldrich, Steinheim, Germany), sodium borohydride (SigmaAldrich, Steinheim, Germany), silver nitrate $99.9 \%$ (PlatLab, São Paulo, Brazil), sodium hydroxide (Synth, São Paulo, Brazil), potassium hexacyanoferrate(II) and potassium hexacyanoferrate(III) (Sigma-Aldrich, Steinheim, Germany) and potassium chloride (Sigma-Aldrich, Steinheim, Germany).

Deionized water used throughout this work was obtained from a MilliQ Millipore system with $18.2 \mathrm{M} \Omega \mathrm{cm}$ resistivity (Millipore, Burlington, USA). Graphite powder and mineral oil were obtained from Sigma-Aldrich (Steinheim, Germany). A $5 \mathrm{mmol} \mathrm{L}^{-1}\left[\mathrm{Fe}(\mathrm{CN})_{6}\right]^{3-/ 4-}$ was prepared in $\mathrm{KCl} 100 \mathrm{mmol} \mathrm{L}^{-1}$ by the dissolution of the respective salts in deionized water. Phosphate buffer saline (PBS) was purchased from Sigma (Steinheim, Germany) and prepared as suggested by the vendor (a tablet in $250 \mathrm{~mL}$ deionized water). Sodium thiocyanate was purchased from Sigma-Aldrich (Steinheim, Germany). A stock solution of $10 \mathrm{mmol} \mathrm{L}^{-1}$ was prepared from the salt in deionized water and diluted as necessary.

\subsection{D-printing and device design}

We designed the device and exported to.stl format using Fusion 360 (Autodesk, San Rafael, USA) and then transferred to Simplify3D (Cincinnati, USA) which was used to convert to.gcode format and to control an FDM-based 3D-printer model Sethi3D S3 (Sethi3D, Campinas, Brazil). The printer was equipped with a $0.2 \mathrm{~mm}$ brass nozzle, where 1.75 mm PLA (UP3D, São Paulo, Brazil) was used as the filament to create the device.

The microfluidic device consisted of a set of channels in serpentine (Fig. 1), where three inlets were used to insert the solutions and one was used to collect the synthesized nanoparticles.

\subsection{Synthesis procedure and characterization}

We synthesized the AuNPs based on our previous publication with some modifications [2]. Here, the following reactant concentrations were used: $38.8 \mathrm{mmol} \mathrm{L}^{-1}$ trisodium citrate $\left(\mathrm{Na}_{3} \mathrm{Ct}\right)$ and $1 \mathrm{mmol} \mathrm{L}^{-1} \mathrm{HAuCl}_{4}$, while the system temperature was kept at $90^{\circ} \mathrm{C}$, and the flow 


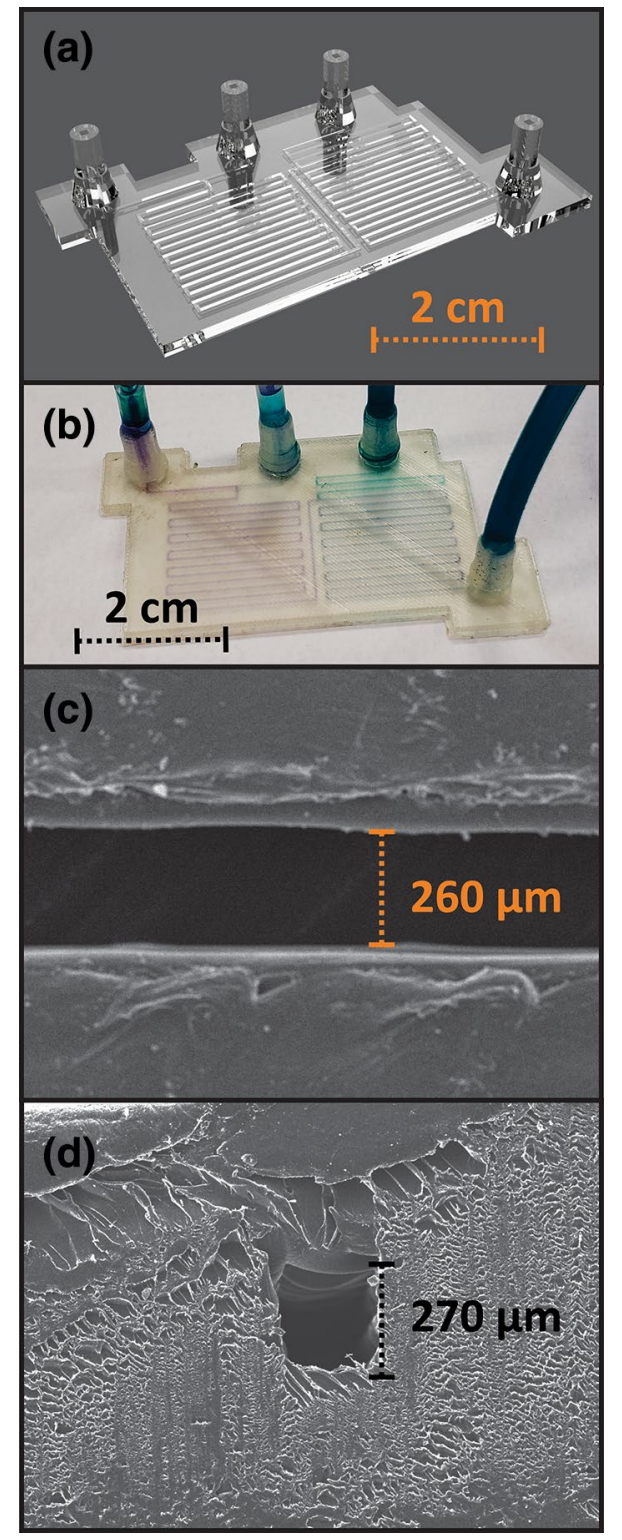

Fig. 1 a Rendering of the CAD object used to print the microfluidic device; $\mathbf{b}$ photograph of the actual 3D-printed device filled with food colorant to improve contrast; c top-view SEM of a channel in the 3D-printed device; and $\mathbf{d}$ cross-sectional view of a channel in the 3D-printed device. Scale bars are indicated in each figure

rate of the reactants was kept at $100 \mu \mathrm{L} \mathrm{min}{ }^{-1}$. After the AuNPs were synthesized, we collected them at a clean centrifuge tube to further modification.

These AuNPs were then diluted ten times and used as seed to formation of core-shell nanoparticles, using a solution of $1 \mathrm{mmol} \mathrm{L}^{-1} \mathrm{NaBH}_{4}$ in $3 \mathrm{mmol} \mathrm{L}^{-1} \mathrm{NaOH}$ as the reductant and $0.75 \mathrm{mmol} \mathrm{L}^{-1} \mathrm{AgNO}_{3}$ as the silver salt to produce the shells. We controlled the flow of the reactants entering the microfluidic device using two Harvard Elite13 syringe pumps (Harvard Apparatus,
Holliston, USA), where we kept the solutions in 3-mL plastic syringes (Becton Dickinson, Franklyn Lakes, USA), driven into the microfluidic device by means of PTFE tubing with $0.3 \mathrm{~mm}$ internal diameter held in place by silicon tubing (Cole Parmer, Vernon Hills, USA).

Shortly after synthesis, the core-shell nanoparticles formed were analyzed by means of UV-Vis spectrometry and were kept refrigerated $\left(4^{\circ} \mathrm{C}\right)$ until measured by transmission electron microscopy (TEM) in a Libra 120 (Carl Zeiss, Oberkochen, Germany), where the samples were put onto carbon films deposited in copper grids with 400 mesh (TedPella, Redding, USA).

\subsection{Carbon paste electrode fabrication, modification and electrochemical analysis}

The carbon paste was produced by mixing 80:20 parts (wt.) of graphite powder and mineral oil, respectively, until a smooth paste was formed. Then, we built the carbon paste electrode (CPE) inserting the graphite paste into a pipette tip and using a copper wire as electrical contact. To perform the modification of the electrode, $40 \mu \mathrm{L}$ of Au@Ag nanoparticles solution $\left(0.0125 \mu \mathrm{mol} \mathrm{L}^{-1}\right)$ was drop-casted onto the CPE surface $\left(14.6 \mathrm{~mm}^{2}\right)$ and left to dry under a gentle nitrogen flow, thus forming the modified electrode (CPE/Au@Ag).

The electrochemical analysis was performed using a three-electrode system, consisting of the CPE or CPE/ Au@Ag as working electrode, a Pt wire as auxiliary electrode and $\mathrm{Ag} / \mathrm{AgCl}(\mathrm{KCl}$ sat.) as reference electrode using $\mathrm{KCl} 0.1 \mathrm{mmol} \mathrm{L}^{-1}$ or PBS $10 \mathrm{mmol} \mathrm{L}^{-1} \mathrm{pH} 7.4$ as the supporting electrolyte, depending on the analyte. Electrochemical impedance spectroscopy (EIS) was performed using an Autolab PGSTAT128N potentiostat (Metrohm, Herisau, Switzerland) equipped with the FRA32M module. The potential of half-wave applied was $0.35 \mathrm{~V}$ for $\left[\mathrm{Fe}(\mathrm{CN})_{6}\right]^{3-/ 4-}$ system and $1.1 \mathrm{~V}$ for thiocyanate ions. The frequencies were scanned from 1 to $10,000 \mathrm{~Hz}$ at ten necessary points in the Nova 2.0 software (Metrohm, Herisau, Switzerland) according to frequencies per decade. The fitting of the semicircle to calculate the charge transfer resistance $\left(R_{\mathrm{ct}}\right)$ was done by marking the points to Randles circuit. Other electrochemical measurements were taken using a PalmSens potentiostat (PalmSens BV, Houten, The Netherlands).

We measured thiocyanate by means of square wave voltammetry (SWV), using a $10 \mathrm{mmol} \mathrm{L}^{-1}$ stock solution diluted as necessary in $10 \mathrm{~mL}$ of $10 \mathrm{mmol} \mathrm{L}^{-1}$ phosphate buffer saline $\mathrm{pH}$ 7.4. The SWV measurements were taken from -0.5 to $+1.5 \mathrm{~V}$, using a step potential of $10 \mathrm{mV}$, an amplitude of $25 \mathrm{mV}$ and a frequency of $30 \mathrm{~Hz}$. 


\section{Results and discussion}

\subsection{D-printed FDM-based microfluidic device}

The 3D-printed microfluidic device used to synthesize the core-shell nanoparticles consisted of a single plastic part (total width $\times$ length $\times$ height $=57 \times 65 \times 12 \mathrm{~mm}$, including the connections) printed with no pauses with PLA. Three inlets and one outlet connected by a serpentine channel were used to deliver the gold nanoparticle seeds, the reductant solution and the silver salt solution, where the first two would be mixed first and then the third would react latter (Figure S1, Supplementary Information) to produce the silver shell. The first reaction portion consisted of a single channel with 13 turns with an overall length of $290 \mathrm{~mm}$ with designed widths and heights of $500 \mu \mathrm{m}$, and the second reaction portion was identical to the first in all dimensions. Figure 1a shows the CAD rendering of the proposed microfluidic device, where Fig. $1 \mathrm{~b}$ shows the actual device filled with food colorant to enhance contrast. The two different reaction sections were used in order to mix the gold nanoparticle seeds with the reductant solution first, then following reduction of silver ions in the second section, as will be discussed later.

After successfully printing the microfluidic device, we evaluated its quality by means of SEM, as shown in Fig. 1c-d, which display the SEM images of an open channel and the transversal profile of the channels. We designed the proposed channels to have width and heights of $500 \mu \mathrm{m}$, but SEM images presented channels of $260 \mu \mathrm{m}$, approximately, reiterating the results of our previous publication [13]. Although channels with similar dimensions have been proposed using masters for molding materials such as PDMS, they often require a multi-step procedure, in which the mold must be removed and the object must be attached to another part to be sealed [26]. Additionally, mold replication only allows the preparation of 2D structures. In our case, the object is printed in a single step, allowing for higher throughput as several devices can be printed at the same time at the printer bed and be ready to use after, since FDM-based 3D-printed objects do not require post manipulation.

We should note that the nozzle used in our printer is $0.2 \mathrm{~mm}$, whereas the default size for most FDM printers is $0.4 \mathrm{~mm}$, leading to higher-resolution and smaller channel sizes, at the expense of printing time. In our case, the device took approximately $2 \mathrm{~h}$ to print using the configurations presented in Table S1 (Supplementary Information). The channel geometry used in the proposed device is supposed to be simple, acting as a simple mixer between the reactants. As seen in Fig. 1d, the deposition of the layers is not exactly aligned due to 3D-printing technique used. However, FDM excels at being the lowest cost 3D printer technique to purchase and maintain, especially because of the filaments used. In our case, we employed PLA because it is readily available and because the natural filament presents some degree of transparency, which allows the verification of fouling inside the microfluidic device. Moreover, the final synthesis of the core-shell nanoparticles was carried out at room temperature, which allows the use of PLA, since it is less heat resistant than acrylonitrile butadiene styrene (ABS) but also less prone to printing failures due to lower printing temperature and shrinkage. Moreover, the cost regarding FDM-based 3D-printed devices is overall low than any other 3D-printing technique and more conventional microfabrication protocols [27], mainly due to low price of the printer itself (starting at 300 US dollars) and the filament used (starting at 20 US dollars per kilogram). In our case, the device printed weights around $5 \mathrm{~g}$, meaning a price per device around 0.10 US dollars.

\subsection{Synthesis and characterization of core-shell Au@Ag nanoparticles}

The synthesis of the gold nanoparticle seeds was based on our previous publication [2] with some modifications as to obtain a better size dispersion and shape uniformity, as seen in Figure S2 (Supplementary Information), where the TEM image of the sample is displayed. To synthesize the core-shell nanoparticles, we used AuNPs as seeds where the silver shell would form around. This is based on a seed growth mechanism, where the nanoparticles act as centers of growth to a new metal reducing in the reaction media $[15,19,28,29]$.

We tested different reactions in order to obtain the core-shell nanoparticles, based on different reductants (trisodium citrate, ascorbic acid and sodium borohydride). However, the observed UV-Vis extinction spectra for both trisodium citrate and ascorbic acid reactions did not present a blue shift in the peak indicating the production of core-shell nanoparticles. The incompleteness could be due to insufficient reaction between the silver salt and the reductant in the proposed microfluidic device due to its geometry or the low temperature used for the synthesis [30]. In either case, changing the device to work in these conditions would be troublesome, whereas using a stronger reductant, e.g., $\mathrm{NaBH}_{4}$ solution, would facilitate the reaction since no heating is required, and the reaction could be carried out at room temperature [31].

Hence, we changed the reductant to sodium borohydride in order to improve the reaction speed and to remove the requirement of heating the microfluidic 
system. We let all reactants reach room temperature $\left(23^{\circ} \mathrm{C}\right)$ prior use. A syringe pump was used to insert two solutions contained in a plastic syringe into the microfluidic device: an AuNPs seed solution $\left(0.050 \mathrm{mmol} \mathrm{L}^{-1}\right)$ in one inlet and $\mathrm{NaBH}_{4}$ solution in $\mathrm{NaOH}$ in the other inlet $\left(1 \mathrm{mmol} \mathrm{L}^{-1}\right.$ in $\left.2.25 \mathrm{mmol} \mathrm{L}^{-1}\right)$. This solution was prepared in $\mathrm{NaOH}$ to prevent the decomposition of the reductant into hydrogen, leading to bubbles inside the syringe that could lead to fluctuations in flow rate. Then, after mixing, another syringe pump controlled the insertion of $\mathrm{AgNO}_{3}$ solution $\left(0.750 \mathrm{mmol} \mathrm{L}^{-1}\right)$ into the other inlet. The reaction then took place inside the second set of serpentine channels and was collected in the outlet.

In the case of our device, we changed the flow proportion to keep the AuNPs flow rate constant $\left(50 \mu \mathrm{L} \mathrm{min}^{-1}\right)$ and to increase the $\mathrm{AgNO}_{3}$ and $\mathrm{NaBH}_{4}$ equally, this way increasing the amount of silver in the system. We changed the parameters to obtain three samples based on different flow rates of silver and $\mathrm{NaBH}_{4}$ solutions: (I) $50 \mu \mathrm{L} \mathrm{min}{ }^{-1}$; (II) $100 \mu \mathrm{L} \mathrm{min}{ }^{-1}$, and (III) $150 \mu \mathrm{L} \mathrm{min}$. UV-Vis spectra were recorded for all samples to evaluate the completeness of reaction (Fig. 2a). For core-shell systems, it is possible to note a blue shift on the surface plasmon resonance (SPR) of the metallic core (AuNPs) toward that of the shell (Ag), which is seen in the UV-Vis spectra from 542 to $450 \mathrm{~nm}$ as the silver concentration is increased (Fig. 2a, Samples I and II). However, when the concentration of silver salt is further increased, another band appears around $700 \mathrm{~nm}$, which is known to be particles with increased sizes that can eventually coalesce together, leading to precipitation of elementary silver (Fig. 2a, Sample III). This change in the SPR can also be observed in the color from the nanoparticle's solution
(Fig. 2b), either for the AuNPs seeds, where a reddish color is predominant, to that of core-shell, where a yellowish color appears. An orange color associated with two bands appearing in the UV-Vis spectra indicates the presence of larger nanoparticles, which could eventually coalesce and precipitate.

We characterized Sample I by means of TEM to further understand the microfluidic synthesis, where an image is displayed in Fig. 2c (further images are displayed in Figure S3 and SEM-EDX analysis in Figure S4, Supplementary Information). The contrast between the shell and core is an indicator of the successful reaction. In addition, the mean diameter of the core-shell Au@Ag nanoparticles was found to be $23 \pm 5 \mathrm{~nm}$, whereas the AuNPs seeds had sizes of $21 \pm 4 \mathrm{~nm}$, as indicated in Fig. $2 \mathrm{~d}$. These values are significatively different at $95 \%$ confidence interval ( $t$ test, $p=0.003$ ), which indicate that a silver shell is indeed formed around the seeds.

Low-cost microfluidic devices, such as FDM-based 3D-printed ones, still do not present optimal channel smoothness due to the inherent layer-by-layer deposition process. However, ease of fabrication, manipulation toward improved designs and cost outweigh the disadvantages [32]. Although not discussed in the present paper, good particle size and distribution has been previous obtained for an FDM-based 3D-printed microfluidic device in the synthesis of single metal nanoparticles. Here, we can observe the formation of larger nanoparticles sizes, which could arise from the heterogeneous deposition of silver around the gold cores. We also noted the presence of silver nanoparticles in the media, but we believe this effect could be lowered by choosing the appropriate parameters, such as flow rate and temperature.

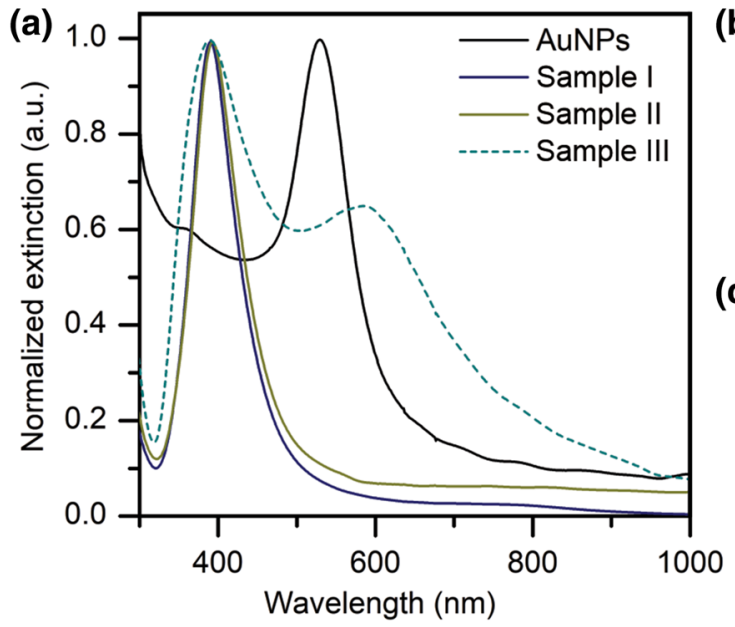

Fig. 2 a Extinction spectra for samples prepared using different flow rate proportions between AuNPs and $\mathrm{AgNO}_{3} ; \mathbf{b}$ photographs of the actual solutions of the nanoparticles after synthesis; c TEM (b)

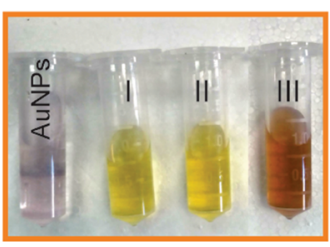

(c)
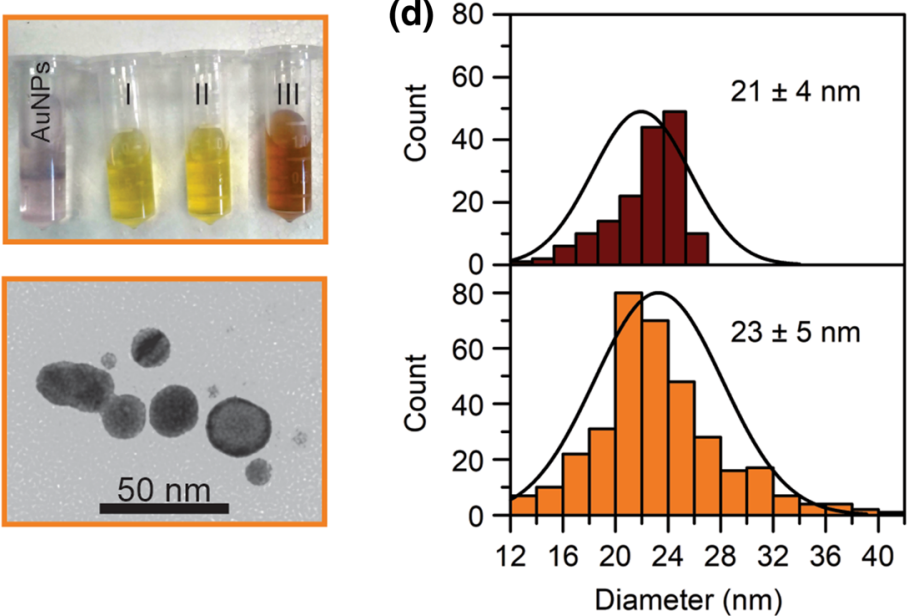

image of core-shell nanoparticles from Sample I; and $\mathbf{d}$ size distribution and mean size \pm standard deviation for both gold nanoparticles and core-shell Au@Ag nanoparticles measured from Sample I 
Several papers dealt with the synthesis of metallic core-shell nanoparticles using more conventional materials or using multi-step fabrication protocols. Knauer et al. [33] used PTFE tubings and PEEK connections to create a coil reactor to synthesize 20-nm-diameter Au@Ag core-shell nanoparticles. The method used has a medium cost due to the tubing used but require manual assembly by the operator and is not fully microfabricated. Sebastián and Jensen [34] used an etched silicon and glass to create a microfluidic device that synthesized several nanoparticles, including Ag@Pd core-shell. Regarding costs, this microfluidic device requires expensive material, a skilled operator and a clean room to be assembled, although the channel quality obtained is optimal. Sachdev et al. [35] used an etched glass microfluidic devices to perform the synthesis of $\mathrm{FeO}_{3} @ \mathrm{Au}$ core-shell nanoparticles with diameter around $12 \mathrm{~nm}$. The microfluidic device was commercially obtained, meaning it was created in a controlled manner, with good channel quality at the expense of cost. To sum up, despite being conventionally prepared in other microfluidic device that use conventional and more expensive materials, we were successful in obtaining core-shell nanoparticles with sizes similar to those of more conventional fabrication methods, using a microfluidic device that is created in a single step and has a very low cost of 0.10 US dollars per device.

\subsection{Use of the synthesized core-shell nanoparticles as modifier to a carbon paste electrode}

To demonstrate the applicability of the nanoparticles synthesized in this study, we used them on the modification of a simple carbon paste electrode, as it is shown that core-shell nanoparticles can enhance the electrocatalytic capability for the electrochemical processes occurring at the electrode surface [36]. The electrode was fabricated as described in Materials and Methods section, and the modification of the working electrode was based on a simple
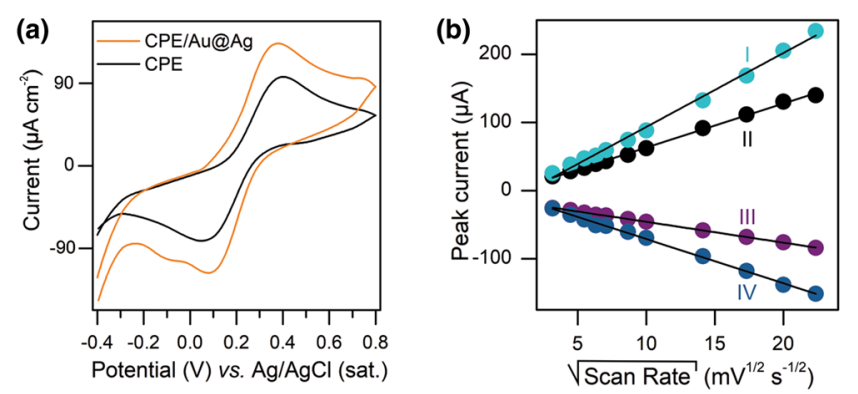

Fig. 3 a Cyclic voltammograms for both CPE and CPE/Au@Ag electrodes at $100 \mathrm{mV} \mathrm{s}^{-1}$; b peak current dependency on the square root of the scan rate for modified (I, anodic and IV, cathodic) and unmodified (II, anodic and III, cathodic) electrodes; c EIS for unmod- drop-casting method, where $40 \mu \mathrm{L}$ of Au@Ag nanoparticles suspension (Sample I) was deposited on the electrode surface and left to dry under nitrogen atmosphere.

The modified electrode was characterized by means of cyclic voltammetry using the electrochemical probe of $5 \mathrm{mmol} \mathrm{L}^{-1}\left[\mathrm{Fe}(\mathrm{CN})_{6}\right]^{3-/ 4-}$ in $100 \mathrm{mmol} \mathrm{L}^{-1} \mathrm{KCl}$ electrolyte. Figure 3a displays a cyclic voltammogram of the probe solution for both CPE and CPE/Au@Ag, where an increase in both anodic and cathodic current peaks is observed for the latter, probably due to the increase on the electrode active surface area. Also, the decrease on the separation of the anodic peak potential $\left(E_{\mathrm{pa}}\right)$ and cathodic peak potential $\left(E_{\mathrm{pc}}\right)$ indicates a better electron transfer with increased electrode kinetic. To calculate the electrochemical active area of the electrodes, we employed the Randles-Sevcik equation [37], which allows confirmation that the electrochemical process is diffusion-controlled, and no adsorption is occurring on the electrode surface. In our case, this equation can be used to confirm that no adsorption is occurring on the proposed modified electrode. The results are displayed in Fig. 3b, where we notice an increase on the slopes for both anodic (I vs. II) and cathodic (IV vs. III) peak currents at different scan rates for CPE/Au@Ag. The relationship between the square root of the scan rate against the peak current is linear, as expected for a diffusion-controlled electrochemical process. The active surface area calculated for CPE and CPE/Au@Ag was 14.6 and $22.9 \mathrm{~mm}^{2}$, respectively, in agreement with increased peak currents for the modified electrode.

We also performed electrochemical impedance spectroscopy (EIS) in both CPE and CPE/Au@Ag electrode, ranging from 1 to $10,000 \mathrm{~Hz}$ in a $5 \mathrm{mmol} \mathrm{L}^{-1}$ redox probe of $\left[\mathrm{Fe}(\mathrm{CN})_{6}\right]^{3-/ 4-}$ on $100 \mathrm{mmol} \mathrm{L}^{-1} \mathrm{KCl}$ supporting electrolyte. The Nyquist plots are shown in Fig. 3c, where the fitted electrochemical data show that the charge transfer resistance $\left(R_{\mathrm{ct}}\right)$, is reduced from 17.5 to $11.8 \mathrm{k} \Omega$ for bare $\mathrm{CPE}$ and CPE/Au@Ag, respectively. As obtained by cyclic voltammetry, the decreased $R_{\mathrm{ct}}$ value for CPE/Au@Ag can
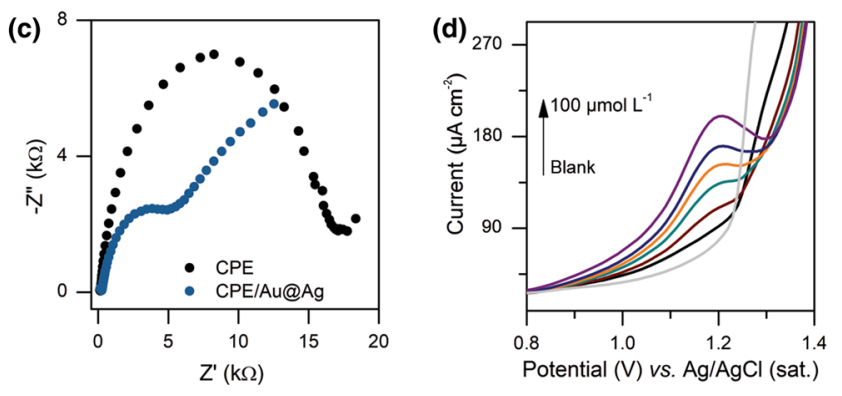

ified and modified electrode; and $\mathbf{d}$ voltammetric profile of thiocyanate ions at increasing concentrations (blank, 4.0, 8.0, 12.0, 25.0, 50.0 and $100 \mu \mathrm{mol} \mathrm{L}^{-1}$ ). Further experimental details are described in the text 
be explained by the enhanced performance of electron transfer at metallic surfaces compared to CPE.

The CPE/Au@Ag was used on the sensing of thiocyanate ions based on a reaction between the silver shell and the thiocyanate ions. In this case, the metallic silver shell is oxidized to silver ions which form a complex with the thiocyanate ions. This complex is then oxidized, giving a corresponding anodic peak [38]. The electrochemical profile for square wave voltammetry of $\mathrm{SCN}^{-}$indicates the presence of an anodic peak at around $+1.2 \mathrm{~V}$, which increases linearly from a blank and $\mathrm{SCN}^{-}$solutions at concentrations ranging from 4 to $100 \mu \mathrm{mol} \mathrm{L}^{-1}$ (Fig. 3d and Figure S5, Supplementary Information). We also performed an EIS measurements in both CPE and CPE/Au@Ag using the thiocyanate ions as the electrochemical probe, where the $R_{\mathrm{ct}}$ decreased from 7.9 to $2.2 \mathrm{k} \Omega$, respectively, indicating an enhanced electrocatalytic activity for the analyte (Figure S6, Supplementary Information). Since other nanoparticles have already been applied as electrode modifiers $[39,40]$, we believe that the incorporation of the Au@ Ag nanoparticles in CPE could be used an electrochemical sensor for thiocyanate ions in biological fluids such as saliva and urine.

\section{Conclusions}

To sum up, as a proof-of-concept, we demonstrated that core-shell nanoparticles can be synthesized inside a microfluidic chip that presents low-cost and low fabrication time, using the most widespread 3D-printing technique, fused deposition modeling. Using gold nanoparticles synthesized in a microfluidic chip as seeds, we created a silver shell using sodium borohydride as the reductant, eliminating the use of a heat source to complete the reaction. We used syringe pumps to control the reactants flow inside the microfluidic chip, to a point that too much silver salt leads to coalescence of metallic silver. We confirmed the core-shell formation using spectroscopic and electron microscopy techniques. The prepared core-shell nanoparticles were then incorporated in the modification of a carbon paste electrode to voltammetric sensing of thiocyanate ions.

Acknowledgements We would like to thank Coordenação de Aperfeiçoamento de Pessoal do Ensino Superior (CAPES, Finance Code 001), National Council for Scientific and Technological Development (CNPq, 140545/2017-4), São Paulo Research Foundation (FAPESP, 2018/13496-8, 2018/06478-3) and INCTBio for financial support and scholarships. We would also like to thank Douglas Silva that acquired the transmission electron microscopy images, which were supported through INCT/INOMAT (National Institute for Complex Functional Materials) financed by FAPESP (2014/50906-9) and CNPq (465452/2014-0)

\section{Compliance with ethical standards}

Conflict of interest All authors declare that they have no conflict of interest.

\section{References}

1. Boken J, Kumar D, Dalela S (2015) Synthesis of nanoparticles for plasmonics applications: A microfluidic approach. Synth React Inorganic, Met Nano-Metal Chem 45:1211-1223. https://doi. org/10.1080/15533174.2014.900798

2. Bressan LP, Robles-Najar J, Adamo CB et al (2019) 3D-printed microfluidic device for the synthesis of silver and gold nanoparticles. Microchem J 146:1083-1089. https://doi.org/10.1016/j. microc.2019.02.043

3. Makgwane PR, Ray SS (2014) Synthesis of nanomaterials by continuous-flow microfluidics: a review. J Nanosci Nanotechnol 14:1338-1363. https://doi.org/10.1166/jnn.2014.9129

4. Martins JP, Torrieri G, Santos HA (2018) The importance of microfluidics for the preparation of nanoparticles as advanced drug delivery systems. Expert Opin Drug Deliv 15:469-479. https:// doi.org/10.1080/17425247.2018.1446936

5. Song Y, Hormes J, Kumar CSSR (2008) Microfluidic synthesis of nanomaterials. Small 4:698-711. https://doi.org/10.1002/ smll.200701029

6. Wagner J, Tshikudo T, Kohler J (2008) Microfluidic generation of metal nanoparticles by borohydride reduction. Chem Eng J 135:S104-S109. https://doi.org/10.1016/j.cej.2007.07.046

7. Bandulasena MV, Vladisavljević GT, Odunmbaku OG, Benyahia B (2017) Continuous synthesis of PVP stabilized biocompatible gold nanoparticles with a controlled size using a 3D glass capillary microfluidic device. Chem Eng Sci 171:233-243. https://doi. org/10.1016/j.ces.2017.05.035

8. Yeo LY, Chang HC, Chan PPY, Friend JR (2011) Microfluidic devices for bioapplications. Small 7:12-48. https://doi. org/10.1002/smll.201000946

9. Chen Z, Han JY, Shumate L et al (2019) High throughput nanoliposome formation using 3D printed microfluidic flow focusing chips. Adv Mater Technol 4:1-9. https://doi.org/10.1002/ admt.201800511

10. Kwak CH, Kang SM, Jung E et al (2018) Customized microfluidic reactor based on droplet formation for the synthesis of monodispersed silver nanoparticles. J Ind Eng Chem 63:405-410. https://doi.org/10.1016/j.jiec.2018.02.040

11. Tothill AM, Partridge M, James SW, Tatam RP (2017) Fabrication and optimisation of a fused filament 3D-printed microfluidic platform. J Micromech Microeng 27:035018. https://doi. org/10.1088/1361-6439/aa5ae3

12. Weisgrab G, Ovsianikov A, Costa PF (2019) Functional 3D printing for microfluidic chips. Adv Mater Technol 1900275:1900275. https://doi.org/10.1002/admt.201900275

13. Bressan LP, Adamo CB, Quero RF et al (2019) A simple procedure to produce FDM-based 3D-printed microfluidic devices with an integrated PMMA optical window. Anal Methods 11:1014-1020. https://doi.org/10.1039/C8AY02092B

14. Nelson MD, Ramkumar N, Gale BK (2019) Flexible, transparent, sub-100 $\mu \mathrm{m}$ microfluidic channels with fused deposition modeling 3D-printed thermoplastic polyurethane. J Micromech Microeng 29:095010. https://doi.org/10.1088/1361-6439/ab2f2 6

15. Ghosh Chaudhuri R, Paria S (2012) Core/Shell Nanoparticles: Classes, Properties, Synthesis Mechanisms, Characterization, 
and Applications. Chem Rev 112:2373-2433. https://doi. org/10.1021/cr100449n

16. Douglas F, Yañez R, Ros J et al (2008) Silver, gold and the corresponding core shell nanoparticles: synthesis and characterization. J Nanoparticle Res 10:97-106. https://doi.org/10.1007/ s11051-008-9374-3

17. Hao N, Nie Y, Xu Z, Zhang JXJ (2019) Ultrafast microfluidic synthesis of hierarchical triangular silver core-silica shell nanoplatelet toward enhanced cellular internalization. J Colloid Interface Sci 542:370-378. https://doi.org/10.1016/j.jcis.2019.02.021

18. Zheng D, Hu C, Gan T et al (2010) Preparation and application of a novel vanillin sensor based on biosynthesis of $\mathrm{Au}-\mathrm{Ag}$ alloy nanoparticles. Sens Actuat B Chem 148:247-252. https://doi. org/10.1016/j.snb.2010.04.031

19. Calagua A, Alarcon H, Paraguay F, Rodriguez J (2015) Synthesis and characterization of bimetallic gold-silver core-shell nanoparticles: a green approach. Adv Nanoparticles 04:116-121. https://doi.org/10.4236/anp.2015.44013

20. Gomez L, Arruebo M, Sebastian V et al (2012) Facile synthesis of $\mathrm{SiO} 2-$ Au nanoshells in a three-stage microfluidic system. J Mater Chem 22:21420. https://doi.org/10.1039/c2jm34206e

21. Gu T, Zheng C, He F et al (2018) Electrically controlled mass transport into microfluidic droplets from nanodroplet carriers with application in controlled nanoparticle flow synthesis. Lab Chip 18:1330-1340. https://doi.org/10.1039/C8LC00114F

22. Tao S, Yang M, Chen $\mathrm{H}$ et al (2017) Microfluidic synthesis of $\mathrm{Ag} @ \mathrm{Cu}_{2} \mathrm{O}$ core-shell nanoparticles with enhanced photocatalytic activity. J Colloid Interface Sci 486:16-26. https://doi. org/10.1016/j.jcis.2016.09.051

23. Köhler JM, Romanus $H$, Hübner U, Wagner J (2007) Formation of star-like and core-shell AuAg nanoparticles during two- and three-step preparation in batch and in microfluidic systems. J Nanomater 2007:1-7. https://doi.org/10.1155/2007/98134

24. Rossi S, Puglisi A, Benaglia M (2018) Additive manufacturing technologies: 3D printing in organic synthesis. ChemCatChem 10:1512-1525. https://doi.org/10.1002/cctc.201701619

25. Trojanowicz M (2020) Flow chemistry in contemporary chemical sciences: a real variety of its applications. Molecules. https://doi. org/10.3390/molecules 25061434

26. Nielsen AV, Beauchamp MJ, Nordin GP, Woolley AT (2020) 3D printed microfluidics. Annu Rev Anal Chem. https://doi. org/10.1146/annurev-anchem-091619-102649

27. Bhattacharjee N, Urrios A, Kang S, Folch A (2016) The upcoming 3D-printing revolution in microfluidics. Lab Chip 16:1720-1742. https://doi.org/10.1039/C6LC00163G

28. Chang J, Zhang A, Huang Z et al (2019) Monodisperse Au@Ag core-shell nanoprobes with ultrasensitive SERS-activity for rapid identification and Raman imaging of living cancer cells. Talanta 198:45-54. https://doi.org/10.1016/j.talanta.2019.01.085

29. Lu L, Burkey G, Halaciuga I, Goia DV (2013) core-shell gold/silver nanoparticles: Synthesis and optical properties. J Colloid Interface Sci 392:90-95. https://doi.org/10.1016/j.jcis.2012.09.057

30. Xu L, Peng J, Yan M et al (2016) Droplet synthesis of silver nanoparticles by a microfluidic device. Chem Eng Process Process Intensif 102:186-193. https://doi.org/10.1016/j.cep.2016.01.017
31. Lazarus LL, Riche CT, Marin BC et al (2012) Two-phase microfluidic droplet flows of ionic liquids for the synthesis of gold and silver nanoparticles. ACS Appl Mater Interfaces 4:3077-3083. https://doi.org/10.1021/am3004413

32. Nielsen JB, Hanson RL, Almughamsi HM et al (2019) Microfluidics: innovations in materials and their fabrication and functionalization. Anal Chem. https://doi.org/10.1021/acs.analc hem.9b04986

33. Knauer A, Eisenhardt A, Krischok S, Koehler JM (2014) Nanometer precise adjustment of the silver shell thickness during automated $\mathrm{Au}-\mathrm{Ag}$ core-shell nanoparticle synthesis in micro fluid segment sequences. Nanoscale 6:5230. https://doi.org/10.1039/ c3nr06438g

34. Sebastián V, Jensen KF (2016) Nanoengineering a library of metallic nanostructures using a single microfluidic reactor. Nanoscale 8:15288-15295. https://doi.org/10.1039/C6NR0 $4104 \mathrm{C}$

35. Sachdev S, Maugi R, Kirk C et al (2017) Synthesis and assembly of gold and iron oxide particles within an emulsion droplet; facile production of core@shell particles. Colloids Interface Sci Commun 16:14-18. https://doi.org/10.1016/j.colcom.2016.12.005

36. Deroco PB, Melo IG, Silva LSR et al (2018) Carbon black supported Au-Pd core-shell nanoparticles within a dihexadecylphosphate film for the development of hydrazine electrochemical sensor. Sens Actuat B Chem 256:535-542. https://doi. org/10.1016/j.snb.2017.10.107

37. El-Desoky H, Abdel-Galeil M, Khalifa A (2019) Mesoporous SiO2 (SBA-15) modified graphite electrode as highly sensitive sensor for ultra trace level determination of Dapoxetine hydrochloride drug in human plasma. J Electroanal Chem 846:113157. https:// doi.org/10.1016/j.jelechem.2019.05.039

38. Wang G-F, Li M-G, Gao Y-C, Fang B (2004) Amperometric sensor used for determination of thiocyanate with a silver nanoparticles modified electrode. Sensors 4:147-155. https://doi. org/10.3390/s40900147

39. Afkhami A, Soltani-Felehgari F, Madrakian T (2014) Highly sensitive and selective determination of thiocyanate using gold nanoparticles surface decorated multi-walled carbon nanotubes modified carbon paste electrode. Sens Actuat B Chem 196:467-474. https://doi.org/10.1016/j.snb.2014.01.115

40. Ponnaiah SK, Prakash P, Vellaichamy B et al (2018) Picomolarlevel electrochemical detection of thiocyanate in the saliva samples of smokers and non-smokers of tobacco using carbon dots doped $\mathrm{Fe}_{3} \mathrm{O}_{4}$ nanocomposite embedded on $\mathrm{g}-\mathrm{C}_{3} \mathrm{~N}_{4}$ nanosheets. Electrochim Acta 283:914-921. https://doi.org/10.1016/j.elect acta.2018.07.012

Publisher's Note Springer Nature remains neutral with regard to jurisdictional claims in published maps and institutional affiliations. 